\title{
Functionally graded PbTe-based compound for thermoelectric applications
}

\author{
Z. Dashevsky, M.P. Dariel, and S. Shusterman \\ Department of Materials Engineering, Ben-Gurion University of the Negev
}

\begin{abstract}
The present study summarizes a feasibility study of the influence of the graded indium profile that is set up by the diffusion of indium from an external source, on the transport properties of PbTe crystals. PbTe crystals were grown by Czochralski technique. The penetration profiles of indium, diffusing from an external gaseous source was determined using Seebeck coefficient measurements in PbTe doped preliminary by $\mathrm{Na}$ impurity. The Seebeck coefficient changed a sign as the indium concentration induced a change from $p$-type to $n$-type character. Doping by indium generates deep impurity states lying close to the edge of the conduction band. Electron concentration practically didn't change along $\mathrm{PbTe}<\mathrm{In}>$ crystal while indium concentration changed from $3 \cdot 10^{19}$ to $5 \cdot 10^{20} \mathrm{~cm}^{-3}$. The thermovoltage $V$ of a PbTe crystal in which an In concentration profile had been established was determined up to temperature $\approx 600{ }^{\circ} \mathrm{C}$ (in this case temperature of the cold side was constant $\approx 50{ }^{\circ} \mathrm{C}$ ). It was discovered that $V$ increases linearly with increasing temperature difference. This effect is connected with practically constant value of Seebeck's coefficient in a wide temperature range through stabilization (pinning) of Fermi level by producing a concentration gradient of In impurity in PbTe crystals.
\end{abstract}

Keywords:

Paper received 07.10.99; revised manuscript received 16.12.99; accepted for publication 21.03.00.

\section{Introduction}

In order for thermoelectricity to be an attractive energy conversion alternative, to be able to challenge successfully competing conversion systems and to promote new applications, the dimensionless figure of merit $Z T$ must attain an average minimum value of 1.5. This rationale stands behind the systematic search for advanced thermoelectric materials that display a potential for maximum $Z T$ values. The figure of merit $Z T$, is a function of two dimensionless parameters $\mathrm{ZF}=F(B, \beta)[1]$. The first dimensionless constant is

$B=\xi \mu k_{B}^{2} q K_{l}$,

where $\xi$ is the density of states in the conduction band, $\mu$ is the carrier mobility, $K_{l}$, stands for the phonon part of the thermal conductivity, $k_{B}$ is Boltzmann's constant, $q$ is the electron charge and $T$ is the absolute temperature. It is apparent from Eq.(1) that a promising thermoelectric material is associated with a high value of electrical properties (mobility) and low heat conductivity. Such materials, most likely semiconductors, should display a high density of states (four or more equivalent band minima, $N_{c}$, are necessary [1]). In accordance with these principles, the type IV-VI semiconductor compound PbTe is one of the best thermoelectric materials. The room-temperature mobility in heavily doped $\mathrm{PbTe}$ is higher than that for $\mathrm{Si}$ and $\mathrm{Ge}\left(\mu \approx 2000 \mathrm{~cm}^{2} / \mathrm{Vsec}\right)$, the phonon part of the thermal conductivity is uniquely low $\left(K_{l} \cong 2 \cdot 10^{-2}\right.$ $\mathrm{W} / \mathrm{cmK})$ and the density of states is high $\left(N_{c}=4\right)$.

The second dimensionless constant, $\beta$, is a parameter that stands for the effect of the minority carriers. In the presence of two types of charge carriers (electrons and holes), $\beta=E_{g} / k_{B} T$, where $E_{g}$ is the width of the band gap and determines the bipolar term in the Seebeck coefficient, $S$, and in the thermal conductivity, $K$. The contribution of the minority carriers to the Seebeck coefficient and the thermal conductivity can be expressed in terms of a second dimensionless parameter:

$-\Delta S \sim \mathrm{e}^{-\beta}$ and $\Delta K \sim e^{-\beta}(4+\beta)^{2}$, respectively.

The thermal excitation of minority carriers plays an adverse role in determining the figure of merit. At the operating temperature, the presence of an operating mecha- 


\section{Z. Dashevsky et al.: Functionally graded PbTe-based compound for...}

nism able to reduce or suppress this process by lowering the density of minority carrier is highly desirable. In monolithic thermoelectric materials that are usually considered, the various material parameters along the operating temperature gradient do not display the optimal properties. A higher efficiency than for traditional thermoelectric materials can be expected, if appropriate property profiles are fitted to the temperature gradient. Three approaches can be taken to achieve this goal: (a) by varying the carrier concentration (doping with shallow level impurities), (b) by varying the width of the band-gap (changing of composition), (c) by producing a concentration gradient of impurities that have deep lying levels. Thermoelectric materials built according any of these approaches are so-called FGM (Functionally Graded Materials) and are the focus of much current interest [2]. In the present work we consider a graded material in which the third of these approaches was applied. The strong influence that impurities exert on the electron properties of $\mathrm{PbTe}$ is connected with the deep-lying states that appear as a result of In doping. An indium impurity in $\mathrm{PbTe}$ displays donor-like properties, yet in spite of the high solubility of In in PbTe (20 mol \% In), the original $\mathrm{NaCl}$-type rock-salt structure is retained and the electron concentration does not exceed $n \cong(3-5) \cdot 10^{18} \mathrm{~cm}^{-3}$ In contrast to shallow lying states, deep lying ones consist of wave functions that belong to several bands. Consequently, their energy levels are not connected to any particular band edge $E_{c}$ and $J_{v}$, they may lie in a band as well as in a band-gap. The behavior of In in PbTe was subject of several investigations [1-4]. It was established that:

- At low temperature the In energy level is close to Ec. Its position doesn't depend on the In concentration up to $N_{\text {In }} \approx 3$ at $\%$.

- Each In atom occupying a $\mathrm{Pb}$ lattice site contributes one free electron and two states. Thus, the impurity level is half-filled.

- At low temperature, the Fermi level practically coincides with the impurity level (pinning of the Fermi level).

- The position of the impurity level with respect to $E c$ can be varied by changing the alloy composition e.g. in $\mathrm{Pb}_{1-\mathrm{x}} \mathrm{Sn}_{\mathrm{x}} \mathrm{Te}$ or the temperature.

- Additional doping of $\mathrm{PbTe}<\mathrm{In}>$ with I (one-fold charged donor) or $\mathrm{Na}$ (one-fold charged acceptor) practically doesn't affect the electron concentration until the additional impurity concentration reaches the concentration of In.

\section{Experimental technique}

PbTe crystals doped by acceptor impurity - Na were grown by the Czochralski technique. The large, 25-30 $\mathrm{mm}$ diameter crystals were grown at $2 \mathrm{~cm} / \mathrm{h}$ growth rate and $\delta=0.51 / \mathrm{s}$ rotation speed. Structural characterization was performed using a Rigaku D/MAX-2000 computer controlled diffractometer. Small $4 \times 4 \times 4 \mathrm{~mm}^{3}$ samples were cut from the single crystals and subjected to diffusion doping with In.
The indium diffused into the $\mathrm{PbTe}$ lattice originated from a gaseous phase. According to this approach, indium atoms were transported onto the PbTe crystal surface from an $\mathrm{In}_{4} \mathrm{Te}_{3}$ vapor source, as described below [5]. Using this approach, a constant level of surface In concentration was maintained during the entire diffusion anneal. These anneals were carried out in the 600 to $750{ }^{\circ} \mathrm{C}$ temperature range, for 25 to 200 hour long durations. At the completion of the diffusion anneals, the samples were rapidly quenched to room temperature. During the diffusion anneal the sample was kept in sealed and evacuated 10-12 mm inside diameter and 6-8 cm long quartz ampoules. The ampoule was built at its end with a round reservoir that contained the $\mathrm{In}_{4} \mathrm{Te}_{3}$ powder, used as a source for the gaseous In. The reservoir was connected by a thin neck ( $\sim 2 \mathrm{~mm}$ diameter $)$ to the rest of the ampoule and contained also some $\mathrm{PbTe}$ powder, from the same source as the sample, to maintain the crystal stoichiometry. The evacuated and sealed ampoules were inserted into a horizontal oven that had a zone of uniform temperature longer than the full length of the ampoule.

After doping anneal the samples were examined by optical microscopy for signs of surface damages. The room temperature Seebeck coefficient along the sample was measured at $10 \mu \mathrm{m}$ intervals using a $5 \mu \mathrm{m}$ diameter «hot» probe. The temperature of probe was kept constant within $\pm 0.1{ }^{\circ} \mathrm{C}$. The Hall effect and conductivity were measured over the 100 to 400 temperature range.

\section{Results and discussion}

The variation of the Seebeck coefficients, measured in a direction parallel to the penetration of In by diffusion in a $p$-type PbTe crystal, is shown in Fig. 1. The Seebeck coefficient for the crystal in which no In penetration took place has, as expected, a constant positive value (line 1). The $S$ values for all crystals in which In had diffused (curves 2 to 5) drop at the front-end of the crystal to a large negative value reflecting the effect of the large In addition that over-compensates the presence of the initial $\mathrm{Na}$ acceptors and transforms the initial $p$-type PbTe into a $n$-type $\mathrm{PbTe}$. The values of $S$ as one proceeds into

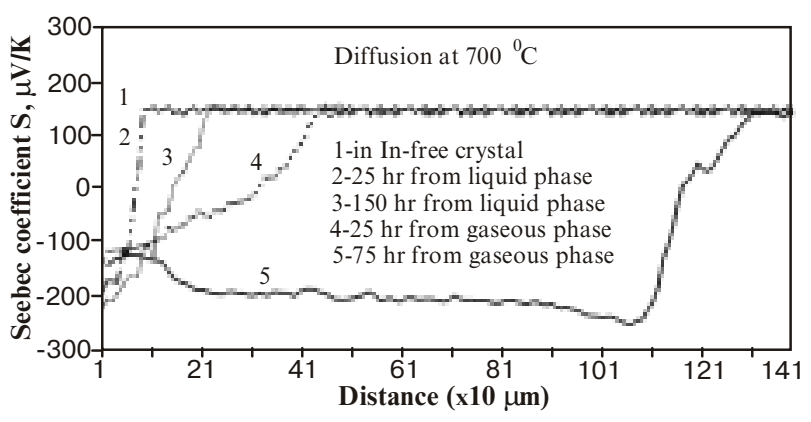

Fig. 1. The variation of the Seebeck coefficient, measured at room temperature along the penetration profile of In into $p$-type PbTe crystal.

$S Q O, 3(2), 2000$ 


\section{Z. Dashevsky et al.: Functionally graded PbTe-based compound for...}

the crystal, increase (algebraically), according to the penetration profile of In, change sign and revert to the positive value, characteristic of In-free PbTe. Clearly, the ability of In, that originates from a gaseous source is significantly more effective than In diffusing from the liquid state, in penetrating the PbTe lattice. The large In concentration gradient that prevails at the interface between the In surface-layer and the PbTe crystal, for samples in which In diffused from the liquid phase, leads possibly to the formation of intermetallic compounds that act as diffusion barriers impeding the penetration of In in the PbTe lattice. Above certain In concentration, the value of $S$ stays at a constant value. According to [6]:

$S=\frac{E_{F}-E_{c}}{q T}-\frac{2 k_{B}}{q}$,

where $E_{c}$ is the edge of conduction band and $E_{F}$ is the Fermi level. The constant value of the Seebeck coefficient, $S$, in samples having reached a certain In concentration indicates that $\left(E_{F}-E_{c}\right)$ remains constant and doesn't depend on the indium concentration This effect is related to the pinning of the Fermi level by the presence In level with respect to the edge of the conduction band, $E_{c}$.

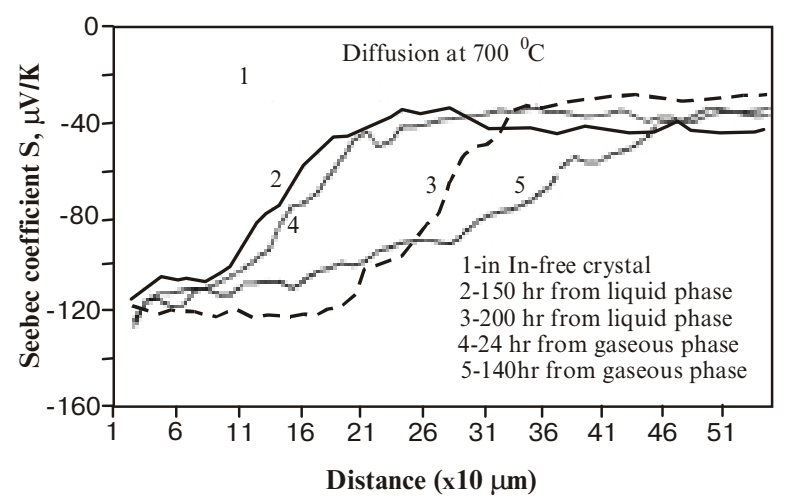

Fig. 2. The variation of the Seebeck coefficient, measured at room temperature along the penetration profile of In into a $n$ type $\mathrm{PbTe}$ crystal.
The variation of the Seebeck coefficient in parallel to the penetration in $n$-type PbTe crystals is shown in Fig. 2. Straight line (1) stands for the measured low value of $S$ in the original crystal doped with shallow level impurities (from the Pbl2 a source) and in which no In had yet been introduced. The large In concentration at the front-end of the crystals in which In has diffused decreases $S$ to large negative values (curves 2 to 5). From the front-end on, the $S$ values increase (algebraically) according to the penetration profiles of In towards the initial low negative value of In-free n-type PbTe. As for $p$-type PbTe crystals, the origin of the In diffusing into the crystals affected to a significant extent its penetration profile. It is also noteworthy that the initial large negative $S$ value in the $n$ type PbTe crystal $(\sim-120 \mathrm{mV} / \mathrm{K})$ is less negative than for the $p$-type crystal $(\sim-200 \mu \mathrm{V} / \mathrm{K})$. This observation can be accounted for in terms of the energy level schemes of initially $n$ and $p$-type PbTe, respectively, in which In has diffused, shown in Fig. 3. The left-side figure shows the energy scheme in an originally $p$-type crystal into which In had diffused.

The presence of In generates a narrow impurity band $\left(E_{\mathrm{In}}\right)$ at room temperature. Some of electrons from the band $E_{\text {In }}$ annihilate the holes that initially were present in the valence band of $p$-type PbTe. The Fermi level $\left(E_{F}\right)$ in the In-containing $p$-type PbTe crystal is located slightly below the In band. In n-type PbTe, even though some of the original electrons of the conduction band dropped into the In-band, the Fermi level is still located in the conduction band. According to Fig.3, clearly $\left(E_{F}-E_{c}\right)$ has a negative value in formerly $p$-type PbTe (Fig.3a), while in initially heavily doped $n$-type $\mathrm{PbTe}$, it has a positive value. Thus, according to eq. 3 , the absolute value of $S$ in the formerly $p$-type will be higher than in the formerly $n$-type crystal.

A $2 \mathrm{~mm}$ thick slice was cut from the $p$-type PbTe sample that had been doped with In originating from the gaseous phase. The portion that was cut coincided with the region in which an gradient was set up as determined by the Seebeck coefficient measurements. The two end-surfaces of the graded crystal were polished and positioned between two flat surfaces. The lower surface was kept at constant $50{ }^{\circ} \mathrm{C}$ and the temperature of the upper surface
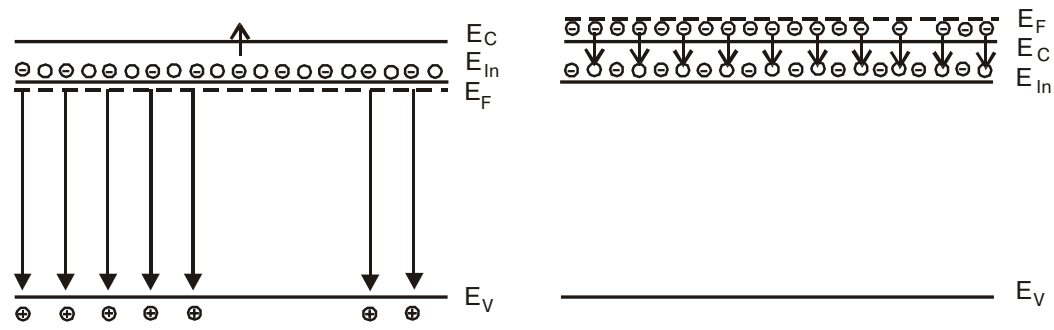

Fig. 3. Schematic band diagram for In-doped PbTe.

a) - in an originally $p$-type PbTe crystal.

b) - in an originally heavily doped $n$-type PbTe crystal. 


\section{Z. Dashevsky et al.: Functionally graded PbTe-based compound for...}

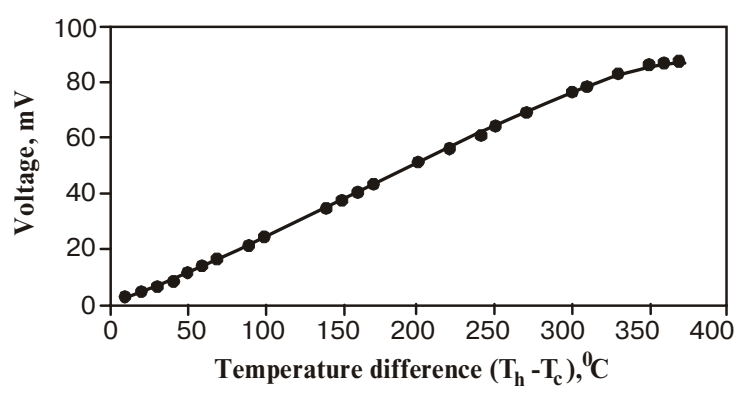

Fig. 4. The thermovoltage of a graded $\mathrm{PbTe}<\mathrm{In}>$ crystal as a function of the temperature difference.

increased up to $430^{\circ} \mathrm{C}$. The measured thermovoltage, $V$, of the graded $n$-doped $\mathrm{PbTe}<\mathrm{In}>$ is shown in Fig. 4 . It is noteworthy that the dependence $V(\Delta T)$ is linear within a close approximation. Thus, the Seebeck coefficient $S=$ $V(\Delta T)$ is practically constant $(S \approx-250 \mu \mathrm{V} / \mathrm{K})$ over a wide temperature range. That behavior of $S(T)$ is very different from that in homogeneous n-type $\mathrm{PbTe}$ (doped only by iodine). The electrons present in the In-generated band annihilate the minority hole carriers whose influence is significant ordinarily at high temperature. Thus, the term $\Delta S$ (Eq. 2) can be neglected and $S$ does not decrease at elevated temperature.

\section{Conclusions}

- In the course of a diffusion anneal at $700{ }^{\circ} \mathrm{C}$ during 75 hours, indium soluted from an external vapour phase generates in $\mathrm{PbTe}$ crystals a penetration profile that extends over a depth of $\approx 1 \mathrm{~mm}$.
- The presence of the deep, In-generated, impurity level (narrow band) lying close to the edge of the conduction band and the annihilation of minority carriers at elevated temperature by the electrons originating in that band allow to achieve an optimal carrier concentration over the 100 to $500{ }^{\circ} \mathrm{C}$ operating temperature range. The Seebeck coefficient stays at a practically constant value over that range.

- The performance of n-type PbTe crystals with a graded structure is improved as compared to that of homogeneous materials. The measured Seebeck coefficient values suggest that the figure of merit in such crystals will attain $Z T \geq 1$ over a temperature range up to $600^{\circ} \mathrm{C}$.

\section{Acknowledgments}

The support of the Israel Science Foundation for the present study within the framework of program 319/97 is gratefully acknowledged.

\section{References}

1. G.D. Mahan, Solid State Physics 51, p. 81 (1997).

2. T. Kajikawa, in Functionally Graded Material 1996, edited by I. Shiota and Y. Miyamoto, (Elsevier Science Publishers, Amsterdam 1997), pp. 475-482.

3. V.I. Kaidanov, Yu. I. Ravich, Soviet Physics Uspekhi 28, p. 31 (1985).

4. T.V. Bocharova, A.N. Veis, Z. M. Dashevsky, V. A. Kotelnikov and R. Yu. Krupitskya, Sov. Phys. Semicond. 15, p. 103 (1981).

5. V. Lyahovitskaya, L. Kaplan, J. Goswami and D. Cahen, Proceedings of the 12-th International Conference on Crystal Growth, Israel, p. 302 (1998).

6. F.J. Hyde, Semiconductors, (Macdonald, London, 1965), p. 265. 